科 学 通 报

\title{
毛白杨叶片 $\mathrm{SO}_{2}$ 急性损伤时呼吸障碍 与低水平化学发光相关性研究 *
}

\section{苏 震 马玉琴 张伸纶 赵克俭} 宗小林 郑雁珍 刘成祥 (中国科学院生物物理研究所, 北京 100101)

\section{关锟词毛白杨叶片、SO$、$ 低水平化学发光}

近年来对 $\mathrm{SO}_{2}$ 损伤机制的研究结果证实, 通过气孔进人叶片的 $\mathrm{SO}_{2}$ 会进行: $\mathrm{SO}_{2}+\mathrm{H}_{2} \mathrm{O} \rightarrow$ $\mathrm{H}_{2} \mathrm{SO}_{3} \rightleftharpoons \mathrm{H}^{+}+\mathrm{HSO}_{3}^{-} \rightleftharpoons 2 \mathrm{H}^{+}+\mathrm{SO}_{3}^{2-}$ 的毒性反应, 致使细胞内 $\mathrm{H}^{+}$的释放, 同时在 $\mathrm{SO}_{3}^{2-}$ 氧化为 $\mathrm{SO}_{4}^{2-}$

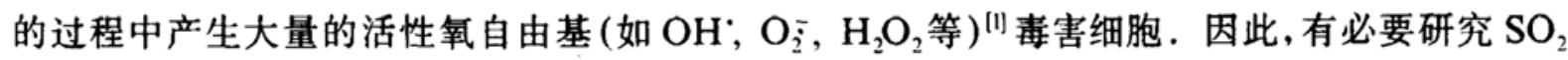
侵人叶片后的损伤机制和过程. 用毛白杨叶片的低水平化学发光 (简称 LCL) 来检测 $\mathrm{SO}_{2}$ 的 污染, 马玉琴等人研究已有良好开端 ${ }^{[2} 31$. 通过薰蒸实验, 发现在 $\mathrm{SO}_{2}$ 急性损伤条件下, 叶片的 LCL 强度和 $\mathrm{SO}_{2}$ 浓度呈正相关. 但目前对 $\mathrm{SO}_{2}$ 所诱发的呼吸障碍方面与其叶片本身发光特 性的关系研究尚少. 本实验着眼于与 $\mathrm{SO}_{2}$ 损伤相关的, 而又引起叶片氧化代谢功能失调的 $\mathrm{OH}^{*}, \mathrm{O}_{i}^{-}$活性氧自由基及 $\mathrm{H}^{+}$作用于毛白杨叶片后呼吸功能的改变及 $\mathrm{LCL}$ 变化, 并找出两者 的相关性.

\section{1 材 料与 方 法}

\section{1 树叶采集}

6-8 月间, 从正常生长的毛白杨树上采摘, 直接放人测量室中进行测量.

\section{2 呼吸强度测定}

红外 $\mathrm{CO}_{2}$ 测定仪, 在避光条件下, 以消除光呼吸可能带来的干扰. 每次测量连枝截取 5 片叶面大小相似的树叶, 重复测量 3 次, 其标准偏差小于 $1 \%$.

\section{3 发光测}

智能化微弱发光测量仪, 可直接测量整片树叶的 LCL. 每次测量 1 片叶片, 至少 5 次重 复,标准偏差小于 $0.5 \%$.

\section{2 结 果与 讨 论}

\section{1 稀 $\mathrm{H}_{2} \mathrm{SO}_{4}$ 浸泡对叶片呼吸速率及低水平化学发光的影响}

用 $0.01 \mathrm{~mol} / \mathrm{L} \mathrm{H}_{2} \mathrm{SO}_{4}$ 浸泡 $30 \mathrm{~s}$ 后树叶呼吸强度的变化, 见图 1-1, 可以看到, 在短时间 (15

1994-02-28 收稿, 1994-05-18 收修改稿.

*国家自然科学基金资助项目. 
$\mathrm{min}$ ) 内, 呼吸强度有一个较块的上升, 随后就渐渐稳定在一个较高的水平上(较浸前大约高 $10 \%$ 左右). 呼吸强度的变化可能是逆境下, 叶片本身对 $\mathrm{SO}_{2}$ 作用的适应, 或是 $\mathrm{H}^{+}$对叶片的

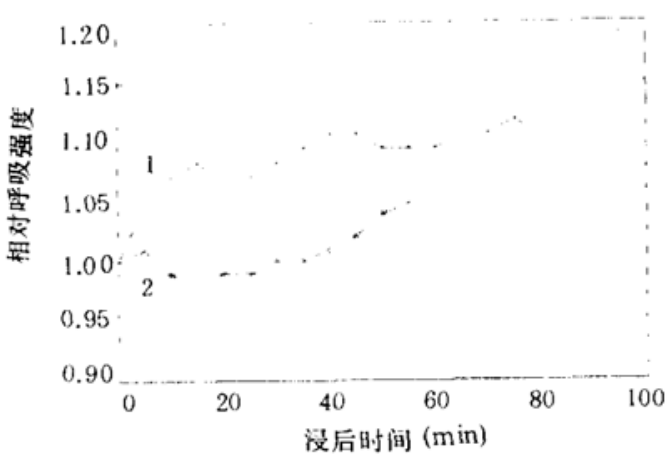

图 1 稀 $\mathrm{H}_{2} \mathrm{SO}_{4}$ 和 $\mathrm{Na}_{2} \mathrm{SO}_{3}$ 溶液浸泡后毛白杨 叶片呼吸强度的变化

1 为稀 $\mathrm{H}_{2} \mathrm{SO}_{4}$ 浸后, 2 为 $\mathrm{Na}_{2} \mathrm{SO}_{3}$ 溶液浸后

表 1 不同因素引起的毛白杨叶片 LCL 和呼吸

强度的化

\begin{tabular}{lcc}
\hline $20 \mathrm{~min}$ 后比较 & LCL 强度增加 (\%) & 呼吸强度增加 $(\%)$ \\
\hline 稀 $\mathrm{H}_{2} \mathrm{SO}_{4}$ & +2.75 & +8 \\
$\mathrm{Na}_{2} \mathrm{SO}_{3}$ 溶液 & -11.01 & -2 \\
Fenton 试剂 & +53.96 & +13 \\
$\mathrm{O}_{2}^{-}$ & -7.86 & -4 \\
\hline
\end{tabular}

损伤, 值得进一步研究. 但从叶片低水平化学发 光角度分析 (见表 1), 发光水平的升高, 代表了 叶片细胞的氧化代谢水平的提高, 这可能是各种 综合损伤作用的一种表现.

从表 1 的数据可以看到, 经稀 $\mathrm{H}_{2} \mathrm{SO}_{4}$ 浸泡 20 $\min$ 后, 由于叶片受到稀 $\mathrm{H}_{2} \mathrm{SO}_{4}$ 伤害而导致呼吸 强度的变化大约升高 $8 \%$ 左右, 与此同时叶片的低水平化学发光值也增加 $2.75 \%$. 两者变化 趋势相似, 由此看来, 叶片的呼吸损伤可由叶片发光强度度量.

另外, 由图 1-1 看出, 随着 $\mathrm{H}_{2} \mathrm{SO}_{4}$ 浸泡时间的延长, 呼吸强度逐渐上升; 相反, 用 $0.01 \mathrm{~mol} / \mathrm{L}$ $\mathrm{Na}_{2} \mathrm{SO}_{3}$ 溶液浸泡后 (图 1-2), 叶片的呼吸强度在 $40 \mathrm{~min}$ 之前变化不大, 只是 $\pm 2 \%$ 的范围内波 动; $40 \mathrm{~min}$ 后呼吸强度略有增加. 可见, 由 $\mathrm{Na}_{2} \mathrm{SO}_{3}$ 解离的 $\mathrm{SO}_{3}^{2-}$, 由于缺少 $\mathrm{H}^{+}$的特定条件, 故 它不能象 $\mathrm{H}_{2} \mathrm{SO}_{4}$ 一样形成酸化环境, 所以, $\mathrm{Na}_{2} \mathrm{SO}_{3}$ 对叶片的呼吸强度影响颇小.

从上述结果可推想, 当 $\mathrm{SO}_{2}$ 侵人叶片组织后, 释放出的 $\mathrm{H}^{+}$对细胞的呼吸作用有较大影 响, 进而在和氧化代谢相关的低水平化学发光上有所体现. 这样, 就看到呼吸强度的增加与 发光强度的增加是一致的.

\section{$2.2 \mathrm{OH} \cdot$ 及 $\mathrm{O}_{i}^{-}$对叶片呼吸强度的影响及其与发光的关系}

(1) $\mathrm{OH}^{\cdot}$ 的作用. Fenton 试剂通过 $\mathrm{Fe}^{2+}+\mathrm{H}_{2} \mathrm{O}_{2} \rightarrow \mathrm{Fe}^{3+}+\mathrm{OH}^{-}+\mathrm{OH}^{*}$ 反应, 可作为 $\mathrm{OH} \cdot$ 源. 图 2 是 Fenton 试剂浸泡后树叶呼吸强度变化曲线. 一经浸泡, 叶片呼吸强度迅速增加, $50 \mathrm{~min}$ 左右出现一个呼吸峰值, 随后呼吸强度逐步下降, 在 $180 \mathrm{~min}$ 时已降到初始值以下, 最终下降 到浸泡前的 $80 \%$ 左右. 该结果与人工熏蒸条件下 $\mathrm{SO}_{2}$ 急性损伤引起的呼吸障碍情况是相一 致的. 提示 $\mathrm{OH} \cdot$ 引发的膜脂质过氧化反应损伤叶片正常的呼吸作用.

与呼吸强度变化相对应, 由 Fenton 试剂浸泡后叶片发光强度的变化由图 3 显示, 先看曲 线 1 , 发光强度有一个激增过程, 然后迅速呈准指数下降, 而当叶片呼吸强度达到高峰时, 叶片 的发光强度已降到接近浸泡前的水平, 表明细胞内的自由基水平已经大大降低了. 这一现象 提示, 叶片通过提高呼吸强度来消除 $\mathrm{OH}$; 这也可以看作是 $\mathrm{OH}^{*}$ 对叶片呼吸有刺激作用的解 释, 同时也可以看作是叶片为减少 $\mathrm{OH}^{*}$ 损伤的一种适应. 为此, 当用几乎没有呼吸强度显示 的近于干枯的叶片, 再浸于 Fenton 试剂后, 由图 3 曲线 2 上可看到发光剧增后, 有一个缓慢 上升的过程, 高峰过后, 才象曲线 1 一样呈准指数规律下降; 需要指出, 该叶片在 $150 \mathrm{~min}$ 内的 发光值高于树叶浸泡前的发光水平. 该结果从又一侧面证实, $\mathrm{OH}^{*}$ 对叶片损伤的重要作用. 


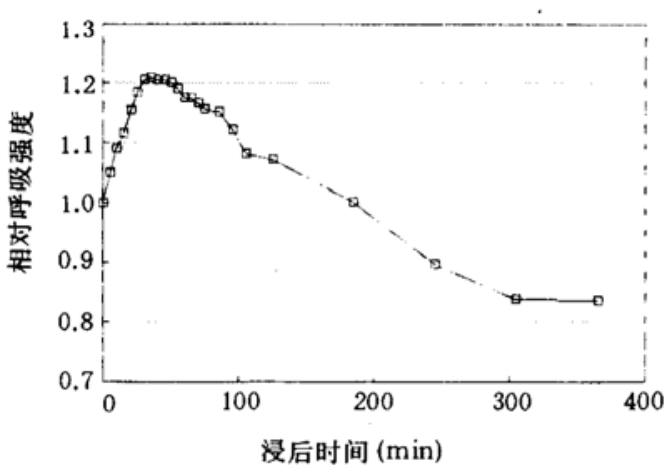

图 2 Fenton 试剂浸泡后毛白杨叶片呼吸强度 的变化

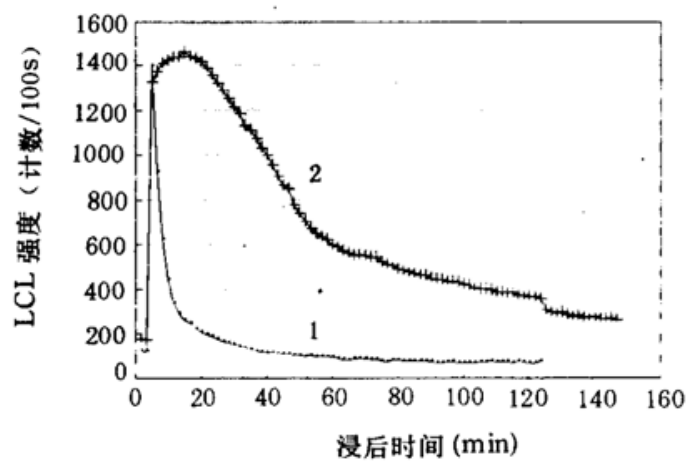

图 3 Fenton 试剂浸泡后毛白杨叶片 LCL 变化 1 为新鲜树叶, 2 为千树叶

这种“闪光发射”现象的出现, 表明 $\mathrm{OH}^{`}$ 对叶片损伤的加剧.

(2) $\mathrm{O}_{i}^{-}$的作用. 在需氧的生物体内到处都存在着 $\mathrm{O}_{2}$, 在 $\mathrm{O}_{2}$ 的存在下, 生物分子甘油酫发 生氧化而使 $\mathrm{O}_{2}$ 接受一个电子, 转变成 $\mathrm{O}_{i}^{-}: \mathrm{O}_{2}$ 甘油郦 $\mathrm{O}_{i}^{-}$. 图 4 显示该甘油醛反应产生的 $\mathrm{O}_{i}^{-}$ 对叶片呼吸作用的影响. 由图清晰可见, 在 0-20 $\min$ 时间间隔内, 叶片的呼吸强度随时间的变化逐 渐减小, 即表 1 示出的呼吸强度出现负增长 $(-4 \%)$, 同时叶片发光强度也下降了 $7.86 \%$, 提示 $\mathrm{O}_{i}^{-}$对叶 片所引起的叶片发光与呼吸作用的变化规律一 致. 和 $\mathrm{OH}^{*}$ 作用不同, $\mathrm{O}_{i}^{-}$所呈现的是对叶片发光 和呼吸作用有双抑制作用。可见,由活性氧自由基 所引起叶片发光和呼吸变化, 应该是 $\mathrm{OH} \cdot$ 和 $\mathrm{O}_{i}^{-} 2$ 种相反作用叠加的结果. 王爱国等人 ${ }^{[4]}$ 认为 $\mathrm{OH}^{*}$ 是导致线粒体膨胀、细胞色素氧化酶活性下降的最 重要因素. 由活性氧引起植物线粒体在结构与功

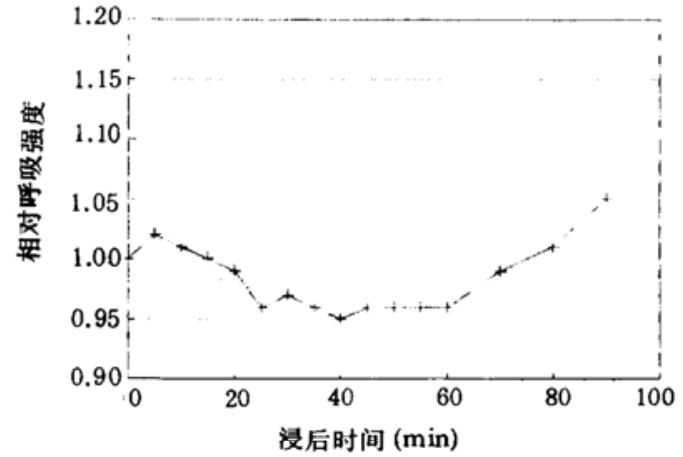

图 $4 \mathrm{O}_{2}^{-}$引起的毛白杨叶片呼吸强度变化 能上的损伤, 又与线粒体膜的脂质过篻化作用有关.

通过稀 $\mathrm{H}_{2} \mathrm{SO}_{4}$ 和 2 种活性氧自由基作用于叶片组织, 测定低水平化学发光强度和呼吸强 度变化的实验, 进行 $\mathrm{SO}_{2}$ 损伤机制的探讨, 是一种新的尝试. 叶片的低水平化学发光的改 变, 不仅远远先于其呼吸强度的变化, 而且发光强度变化显著, 表明用叶片自身的低水平化学 发光的变化作为一种生物物理指标, 监测 $\mathrm{SO}_{2}$ 污染, 评价 $\mathrm{SO}_{2}$ 生物学损伤后果, 方法简便易 行. 从方法学上优于它法, 有一定的实用和推广价值.

\section{考文献}

[1] 钱永常、余叔文, 植物生理学通讯, 1991, 27(5): 326-331.

[2] Ma Yuqin et al., Bull. Environ. Contam. Toxicol., 1992, 49: 906-913.

[3]马端等, 科学通报、1992, 37(24): 2270-2271.

[4]王爱国等, 植物生理学报, 1990, 16(1): 13-18.

[ 5] Abcles, F. B., Ann. Rev. Plant Physiol., 1986, 3: 49-72. 